\title{
REMARKS ON MULTIPLIERS ON CERTAIN FUNCTION SPACES ON GROUPS
}

\author{
HIROSHI YAMAGUCHI \\ (Received 14 February 1979; revised 1 March 1980) \\ Communicated by E. Strzelecki
}

\begin{abstract}
Let $G$ be a LCA group with an algebraically ordered dual $\hat{G}$. Suppose also that the semigroup $P$ of positive elements in $\hat{G}$ is not dense in $\hat{G}$. Subspaces $H_{P}^{s}(G)(1<s<\infty)$ are defined analogous to the Hardy spaces on the circle group, and the question whether every multiplier from $H_{P}^{s}(G)$ into $H \xi(G)$ can be extended to a multiplier from $L^{s}(G)$ into $L^{q}(G)$ is investigated. If we suppose that $s \neq \infty$, then it is shown that such an extension is possible if and only if $(s, q) \in(1, \infty) \times[1, \infty] \cup\{(1, \infty)\}$. (The negative result for $(1,1)$ was obtained in a previous paper.)
\end{abstract}

1980 Mathematics subject classification (Amer. Math. Soc.): 43 A 22.

\section{Introduction}

Let $G$ be a LCA group with the dual group $\hat{G}$. For $1 \leqslant p<\infty L^{p}(G)$ denotes the usual Banach space. Let $M(G)$ be the Banach algebra consisting of all bounded regular measures on $G$. For a subset $E$ of $\hat{G}, L_{E}^{1}(G)\left(M_{E}(G)\right)$ denotes the subspace of $L^{1}(G)(M(G))$ consisting of functions (measures) whose Fourier transforms vanish off $E$. Let $C_{0}(G)$ be the space of all continuous functions on $G$ which vanish at infinity and $C_{c}(G)$ the space of all continuous functions on $G$ which have compact supports, "“' and "' denote the Fourier transform and the inverse Fourier transform respectively. When $\hat{G}$ is algebraically ordered, we define subspaces $H_{P}^{s}(G)$ of $L^{s}(G)(1<s \leqslant \infty)$ which are analogous to the Hardy spaces on the circle group. In a previous paper (Yamaguchi (1980)), the author showed that there exists a multiplier on $H_{P}^{1}(G)$ which can not be represented by convolution with a measure (the compact case is due to Caudry), 
that is there exists a multiplier on $H_{P}^{1}(G)$ which can not be extended to a multiplier on $L^{1}(G)$. In Section 2, if we suppose that $s \neq \infty$, then it is shown that a multiplier from $H_{P}^{s}(G)$ into $H_{P}^{q}(G)$ can be extended to a multiplier from $L^{s}(G)$ into $L^{q}(G)$ whenever $(s, q) \in(1, \infty) \times[1, \infty] \cup\{(1, \infty)\}$.

Next, in Section 3, we show that a linear operator on $H_{P}^{\infty}(G)$ which is continuous with respect to the weak* topology and commutes with translations is given by convolution with a bounded regular measure on $G$. We also construct a multiplier on $H_{p}^{\infty}(G)$ which is not given by convolution with a bounded regular measure on $G$. Let $\beta$ be the canonical map from $C_{0}(G)$ onto $C_{0}(G) / C_{0}(G) \cap H_{(-P)}^{\infty}(G)$. In Section 4, we show that there exists a multiplier $S$ on $C_{0}(G) / C_{0}(G) \cap H_{(-P)}^{\infty}(G)$ such that $\beta \circ S^{\prime} \neq S \circ \beta$ for every multiplier $S^{\prime}$ on $C_{0}(G)$.

Defintion 1.1. Let $\Gamma$ be a LCA group. $\Gamma$ is called an algebraically ordered group if and only if there exists a subsemigroup $P$ of $\Gamma$ with the (AO)-condition, that is (i) $P \cup(-P)=\Gamma$ and (ii) $P \cap(-P)=\{0\}$ (see Doss (1968), p. 257). We do not assume the closedness of $P$.

Let $G$ be a LCA group such that $\hat{G}$ is algebraically ordered. Let $P$ be a subsemigroup of $G$ with the (AO)-condition such that it is not dense in $\hat{G}$. We define $H_{P}(G)(1 \leqslant s \leqslant \infty)$ and $M_{P}^{a}(G)$ as follows:

$$
\begin{gathered}
H_{P}^{1}(G)=\left\{f \in L^{1}(G) ; \hat{f}(\gamma)=0 \text { on } P^{c}\right\}, \\
H_{P}^{\infty}(G)=\left\{f \in L^{\infty}(G) ; \int_{G} f(x) g(x) d x=0 \text { for } g \in H_{P}^{1}(G)\right\}, \\
H_{P}^{s}(G)=\left\{f \in L^{s}(G) ; \hat{f}(\gamma)=0 \text { a.e. on }\left(P^{-}\right)^{c}\right\} \text { for } 1<s \leqslant 2,
\end{gathered}
$$

where $P^{-}$denotes the closure of $P$,

$$
H_{P}^{q}(G)=\left\{f \in L^{q}(G) ; \int_{G} f(x) g(x) d x=0 \text { for every } g \in H_{P}^{q}(G)\right\}
$$

for $2<q<\infty$, where $1 / q+1 / q^{\prime}=1$,

$$
M_{P}^{a}(G)=\left\{\mu \in M(G) ; \hat{\mu}(\gamma)=0 \text { on } P^{c}\right\} \text {. }
$$

REMARK 1.1. (i) If $G=T$ and $P=Z^{+}$(the semigroup of nonnegative integers), $H_{P}^{1}(T)$ is the usual $H^{1}(T)$ and $H_{P}^{\infty}(T)=H_{0}^{\infty}(T) \quad(=\{f \in$ $\left.H^{\infty}(T) ; \hat{f}(0)=0\right\}$ ). If $G=R$ and $P=R^{+}$(the semigroup of nonnegative real numbers), $H_{P}^{1}(R)$ and $H_{P}^{\infty}(R)$ are $H^{1}(R)$ and $H^{\infty}(R)$ respectively.

(ii) Let $G$ be a compact connected abelian group. Then there exists a subsemigroup $P$ of $\hat{G}$ with the (AO)-condition. Let $H^{s}(G)(1<s \leqslant \infty)$ be the 
Hardy spaces defined in (Rudin (1962), 8.1.8, p. 197). Then the following relations are satisfied:

$$
\begin{aligned}
& H_{P}^{s}(G)=H^{s}(G) \text { for } 1 \leqslant s \leqslant 2, \\
& H_{P}^{q}(G)=H_{0}^{q}(G)\left(=\left\{f \in H^{q}(G) ; \hat{f}(0)=0\right\}\right) \text { for } 2<q<\infty .
\end{aligned}
$$

Definition 1.2. Let $A$ and $B$ be subspaces of $L^{p}(G)$ and $L^{q}(G)$ respectively. Let $T$ be a continuous linear operator from $A$ into $B$. $T$ is called a multiplier if $T$ commutes with $\tau_{a}(a \in G)$, where $\tau_{a} f(x)=f(x-a)$.

Doss proved in (Doss (1970), Theorem 2, p. 64) that there exists an analytic projection $U$ from $L^{s}(G)$ onto $H_{P}^{s}(G)$ for $1<s \leqslant 2$. Let $U^{*}$ be the adjoint of $U$. Then, since $U$ is a translation invariant projection, $I-U^{*}$ is also. Hence, for $1<s<\infty$, every multiplier from $H_{P}^{s}(G)$ into $H^{q}(G)(1 \leqslant q \leqslant \infty)$ can be extended to a multiplier from $L^{s}(G)$ into $L^{q}(G)$.

Definition 1.3. For a LCA group $\Gamma$, the coset ring of $\Gamma_{d}$ is the smallest ring of sets consisting of cosets of arbitrary subgroups of $\Gamma$.

Proposition 1.4. Let $P$ be a subsemigroup of $\hat{G}$ with the $(A O)$-condition such that it is not dense in $\hat{G}$. Then $\left(P^{c}\right)^{-}$does not belong to the coset ring of $\hat{G}_{d}$ (the discrete dual of $G$ ).

Proof. By the structure theorem of LCA groups, $\hat{G} \cong R^{n} \oplus F$, where $n$ is a nonnegative integer and $F$ is a LCA group which contains a compact open subgroup $F_{0}$.

Case 1. Suppose $n=0$.

Since $F_{0}$ is compact, $P$ is dense in $F_{0}$ (see Otaki (1977), Lemma 1, p. 307). Since $P$ is not dense in $\hat{G}$ and $\hat{G}=\bigcup\left\{\gamma+F_{0} ; \gamma \in \hat{G}\right\}$, there exists $\gamma_{0} \in \hat{G}$ such that $P$ is not dense in $\gamma_{0}+F_{0}$. By $\left[\gamma_{0}+F_{0}\right]$, we denote the open subgroup of $\hat{G}$ generated by $\gamma_{0}$ and $F_{0}$. Then $\left[\gamma_{0}+F_{0}\right]$ is isomorphic to $Z \oplus F_{0}$, and by (Yamaguchi (1980), Lemma 7), we have $P \cap\left[\gamma_{0}+F_{0}\right] \cong\left\{(n, f) \in Z \oplus F_{0} ; n\right.$ $>0$, or $n=0$ and $\left.f \geqslant_{P} 0\right\}$ (because $P$ is not dense in $\left[\gamma_{0}+F_{0}\right]$ and $P$ is dense in $F_{0}$ ), where ' $\leqslant P$ ' denotes the order on $F_{0}$ induced by $P$. Hence we have $\left(P^{c}\right)^{-} \cap\left[\gamma_{0}+F_{0}\right]=\left\{(n, f) \in Z \oplus F_{0} ; n \leqslant 0\right\}$. Suppose that $\left(P^{c}\right)^{-}$belongs to the coset ring of $\hat{G}_{d}$. Then $\left\{n \gamma_{0} ; n \leqslant 0\right\}$ also belongs to the coset ring of $\hat{G}_{d}$. This is a contradiction.

Case 2. Suppose $n \geqslant 1$.

First we consider the case that $P$ is dense in $R^{n} \oplus F_{0}$. Then, since $P$ is not dense in $\hat{G}$, there exists $\gamma_{0} \in \hat{G}$ such that $P$ is not dense in $\left[\gamma_{0}+R^{n} \oplus F_{0}\right] \simeq Z$ $\oplus R^{n} \oplus F_{0}$ (see Yamaguchi (1980), Lemma 9). Hence we can prove that $\left(P^{c}\right)^{-}$ does not belong to the coset ring of $\hat{G}_{d}$ by the same method in Case 1 . 
Next we consider the case that $P$ is not dense in $R^{n} \oplus F_{0}$. Then $P$ is not dense in $R^{n}$. (because $P$ is dense in $F_{0}$ ). From (Yamaguchi (1980), Lemma 5 and Lemma 10), we may assume that $\left(P^{c}\right)^{-} \cap R^{n} \oplus F_{0}=\left\{\left(x_{1}, x_{2}, \ldots, x_{n}, f\right) \in R^{n}\right.$ $\left.\oplus F_{0} ; x_{1} \geqslant 0\right\}$. Hence $\left(P^{c}\right)^{-}$does not belong to the coset ring of $\hat{G}_{d^{\prime}}$.

Therefore, in each case, $\left(P^{c}\right)^{-}$does not belong to the coset ring of $\hat{G}_{d}$, and the proof is completed.

By Proposition 1.4 and (Birtel (1966), Theorem 3, p. 268), there is no analytic projection from $L^{1}(G)$ onto $H_{P}^{1}(G)$. However the following proposition is satisfied.

Proposition 1.5. Let $G$ be a nondiscrete LCA group whose dual $\hat{G}$ is algebraically ordered. Let $P$ be a subsemigroup of $\hat{G}$ with the $(A O)$-condition such that it is not dense in $\hat{G}$. Then a multiplier from $H_{P}^{1}(G)$ into $H_{P}^{\infty}(G)$ can be extended to a multiplier from $L^{\prime}(G)$ into $L^{\infty}(G)$.

Proof. Let $T$ be a multiplier from $H_{P}^{1}(G)$ into $H_{P}^{\infty}(G)$. For $f \in H_{P}^{1}(G)$, we have

$$
\left\|\tau_{x} T f-T f\right\|_{\infty} \leqslant\|T\|\left\|\tau_{x} f-f\right\|_{1} .
$$

Hence we have $\lim _{x \rightarrow 0}\left\|\tau_{x} T f-T f\right\|_{\infty}=0$. Therefore, $T f$ belongs to $C_{u}(G)$ (the space of all bounded uniformly continuous functions on $G$ ). We define a bounded linear functional $A$ on $H_{P}^{1}(G)$ as follows;

$$
A(f)=T f(0) \text { for } f \in H_{P}^{1}(G) .
$$

By Hahn-Banach's extension theorem, there exists $g \in L^{\infty}(G)$ such that

$$
A(f)=\int_{G} f(-y) g(y) d y \quad \text { for } f \in H_{P}^{1}(G) .
$$

Since $T$ commutes with translations, we have

$$
T f(x)=f * g(x) \text { for } f \in H_{P}^{\prime}(G) \text { and } x \in G .
$$

Therefore $T$ can be extended to a multiplier from $L^{1}(G)$ into $L^{\infty}(G)$.

\section{Multipliers from $H_{P}^{1}(G)$ into $H_{P}^{q}(G)$}

From (Yamaguchi (1980), Theorem 11), there exists a multiplier on $H_{P}^{1}(G)$ which cannot be extended to a multiplier on $L^{1}(G)$. In this section, we prove that there exists a multiplier from $H_{P}^{1}(G)$ into $H_{P}^{q}(G)(1<q<\infty)$ such that it cannot be extended to a multiplier from $L^{1}(G)$ into $L^{q}(G)$. 
THEOREM 2.1. Let $G$ be a nondiscrete LCA group whose dual $\hat{G}$ is algebraically ordered. Let $P$ be a subsemigroup of $\hat{G}$ with the $(A O)$-condition such that it is not dense in $\hat{G}$. Let $q$ be in $[1, \infty)$. Then there exists a multiplier from $H_{P}^{1}(G)$ into $H_{P}^{q}(G)$ such that it can not be extended to a multiplier from $L^{1}(G)$ into $L^{q}(G)$.

Proof. Let $T_{\Phi}$ be the multiplier on $H_{P}^{1}(G)$ constructed in (Yamaguchi (to appear), Theorem 11). We note that $\Phi$ is constructed as follows:

(1) $L_{\text {supp }(\Phi)}^{1}(G) \subset \cap\left\{L^{p}(G) ; 1 \leqslant p<\infty\right\}$,

(2) $P^{-} \cap(-\operatorname{supp}(\Phi))=\varnothing$,

(3) there exist an open set $(0 \in) U$ in $\hat{G}$ with the compact closure, a sequence $\left\{\gamma_{m}\right\}$ in $\hat{G}$ and a nonzero function $\Delta$ in $A(\hat{G})\left(=L^{1}(G)^{\wedge}\right)$ which satisfy the following properties:

(3) $\cup_{1}^{\infty}\left(\gamma_{m}+U\right) \subset P^{0}$ and $\left(\gamma_{m}+U\right) \cap\left(\gamma_{n}+U\right)=\varnothing$ if $m \neq n$,

(3) $\mathrm{II} \Delta \geqslant 0$ and $\operatorname{supp}(U) \subset U$,

(3) $)_{\text {III }} \Phi\left(\gamma+\gamma_{n}\right)=\Delta(\gamma)$ on $U(n=1,2,3, \ldots)$.

First we prove that $T_{\Phi}\left(H_{P}^{1}(G)\right) \subset \cap_{1<s<\infty} H_{P}^{s}(G)$. It is easy to check that $T_{\Phi}\left(H_{P}^{1}(G)\right) \subset \cap_{1 \leqslant s \leqslant 2} H_{P}^{s}(G)$. Let $s$ be in $(2, \infty)$. Let $f$ be a function in $H_{P}^{1}(G)$. Then there exists a sequence $\left\{h_{m}\right\}$ in $H_{P}^{1}(G)$ such that

(4) $\operatorname{supp}\left(\hat{h}_{n}\right)$ has a compact support $(n=1,2, \ldots)$,

(5) $\operatorname{supp}\left(\hat{h_{n}}\right) \subset \operatorname{supp}(\Phi)$,

(6) $\lim _{n \rightarrow \infty}\left\|T_{\Phi} f-h_{n}\right\|_{1}=0$.

Hence, by (1) and (6), we have $\lim _{n \rightarrow \infty}\left\|T_{\Phi} f-h_{n}\right\|_{s}=0$. For each $g \in H_{P}^{q}(G)$ $(1 / s+1 / q=1)$, we have

$$
\begin{aligned}
\int_{G} T_{\Phi} f(x) g(x) d x & =\lim _{n \rightarrow \infty} \int_{G} h_{n}(x) g(x) d x \\
& =\lim _{n \rightarrow \infty} \int_{G} \hat{h}_{n}(-\gamma) \hat{g}(\gamma) d \gamma \\
& =0 \quad \text { (by }(2)) .
\end{aligned}
$$

Thus $T_{\Phi} f$ belongs to $H_{P}^{s}(G)$.

If $T_{\Phi}$ can be extended to a multiplier from $L^{1}(G)$ into $L^{q}(G)(1<q<\infty)$, there exists a function $g$ in $L^{q}(G)$ such that $T_{\Phi} f=f * g$ for $f \in H_{P}^{l}(G)$. But this is impossible by (3) $\sim(3)_{\mathrm{III}}$. Hence $T_{\Phi}$ can not be extended to a multiplier from $L^{\prime}(G)$ into $L^{q}(G)$. This completes the proof.

\section{Multipliers on $H_{P}^{\infty}(G)$}

If $G$ is a compact abelian group whose dual is ordered, each multiplier on $H^{\infty}(G)$ is given by convolution with a bounded regular measure on $G$ (Larsen 
(1971), Theorem 7.3.1, p. 222). In this section we prove that a linear operator on $H_{P}^{\infty}(G)$ which is continuous with respect to the weak* topology and commutes with translations is given by convolution with a bounded regular measure on $G$. Moreover we construct a multiplier on $H_{P}^{\infty}(G)$ which is not given by convolution with a measure.

LEMMA 3.1. Let $G$ be a nondiscrete, noncompact LCA group whose dual $\hat{G}$ is algebraically ordered. Let $P$ be a subsemigroup of $\hat{G}$ with the $(A O)$-condition such that it is not dense in $\hat{G}$. Then, for each neighborhood $V$ of 0 in $\hat{G}, P^{-} \cap V$ has a nonempty interior. Moreover there exists a function $h_{V}$ in $L^{1}(G) \cap H_{P}^{\infty}(G)$ such that $\hat{h}_{V} \geqslant 0, \operatorname{supp}\left(\hat{h}_{V}\right) \subset P^{-} \cap V$ and $\int_{\hat{G}} \hat{h}_{V}(\gamma) d \gamma=1$.

Proof. By the structure theorem of LCA groups, $\hat{G}=R^{n} \oplus F \mathrm{~d}$, where $n$ is a nonnegative integer and $F$ is a LCA group which contains a compact open subgroup $F_{0}$. If $n=0, F_{0}$ is a nontrivial compact open subgroup of $\hat{G}$. Hence, in this case, $P^{-}$contains $F_{0}$ (see Yamaguchi (1980), Lemma 4). Therefore we may assume that $n \geqslant 1$.

Case 1. Suppose $P \cap R^{n}$ is dense in $R^{n}$.

Since $P$ is dense in $F_{0}$, we have $P^{-} \supset R^{n} \oplus F_{0}$. Hence $P^{-} \cap V$ has a nonempty interior. It is easy to construct such a function $h_{V} \in L^{1}(G) \cap$ $H_{P}^{\infty}(G)$.

Case 2. Suppose $P \cap R^{n}$ is not dense in $R^{n}$.

By (Yamaguchi (to appear), Lemma 5), we may assume that $\left(P \cap R^{n}\right)^{0} \supset\{x$ $\left.=\left(x_{1}, x_{2}, \ldots, x_{n}\right) \in R^{n} ; x_{1}>0\right\}$ without loss of generality. Since $P^{-} \supset\{x=$ $\left.\left(x_{1}, x_{2}, \ldots, x_{n}\right) \in R^{n} ; x_{1} \geqslant 0\right\} \times F_{0}, P^{-} \cap V$ has a nonempty interior. In this case, we can construct such a function $h_{V} \in L^{1}(G) \cap H_{P}^{\infty}(G)$. This completes the proof of Lemma 3.1 .

LEMMA 3.2. Let $G$ be a nondiscrete LCA group whose dual $\hat{G}$ is algebraically ordered. Let $P$ be a subsemigroup of $\hat{G}$ with the $(A O)$-condition such that it is not dense in $\hat{G}$. Then $\left(C_{0}(G) \cap H_{P}^{\infty}(G)\right)^{-}=H_{P}^{\infty}(G)$, where 'bar' denotes weak" closure.

Proof. If $G$ is compact, we can prove this lemma easily. Hence we may assume that $G$ is not compact. Let $\gamma_{0}$ be in $P \backslash\{0\}$. For each compact neighborhood $V$ of 0 in $\hat{G}$, by Lemma 3.1, there exists a function $h_{V} \in L^{1}(G) \cap$ $H_{P}^{\infty}(G)$ such that $\hat{h}_{V} \geqslant 0, \operatorname{supp}\left(\hat{h}_{V}\right) \subset V \cap P^{-}$and $\int_{\hat{G}} \hat{h}_{V}(\gamma) d \gamma=1$. We note that $h_{V} \in C_{0}(G)$ and $H_{P}^{\infty}(G)$ is a subalgebra of $L^{\infty}(G)$.

Claim. $\lim _{V} \gamma_{0} h_{V}=\gamma_{0}\left(\right.$ weak $\left.^{*}\right)$. 
Indeed, for $f \in L^{1}(G)$, we have

$$
\begin{aligned}
\left\langle\gamma_{0} h_{V}, f\right\rangle & -\left\langle\gamma_{0}, f\right\rangle=\int_{G}\left(x, \gamma_{0}\right) h_{V}(x) f(-x) d x-\int_{G}\left(x, \gamma_{0}\right) f(-x) d x \\
& =\int_{G}\left(x, \gamma_{0}\right) \int_{\hat{G}} \hat{h}_{V}(\gamma)(x, \gamma) d \gamma f(-x) d x-\int_{\hat{G}} \hat{h}_{\nu}(\gamma) \int_{G}\left(x, \gamma_{0}\right) f(-x) d x d \gamma \\
& =\int_{\hat{G}} \hat{h}_{\nu}(\gamma)\left(\hat{f}\left(\gamma+\gamma_{0}\right)-\hat{f}(\gamma)\right) d d \gamma .
\end{aligned}
$$

Since $\hat{f}(\gamma)$ is uniformly continuous, we have $\lim _{V}\left\langle\gamma_{0} h_{V}, f\right\rangle=\left\langle\gamma_{0}, f\right\rangle$. Hence we have (a) $P \backslash\{0\} \subset\left(C_{0}(G) \cap H_{P}^{\infty}(G)\right)^{-}$. We note that $\left(L^{1}(G) / H_{(-P)}^{1}(G)\right)^{*} \simeq$ $H_{P}^{\infty}(G)$. Suppose that $\left(C_{0}(G) \cap H_{P}(G)\right)^{-} \varsubsetneqq H_{P}^{\infty}(G)$. Then there exists $[f] \in$ $L^{1}(G) / H_{(-P)}^{1}(G)$ and $g \in H_{P}^{\infty}(G) \backslash\left(C_{0}(G) \cap H_{P}^{\infty}(G)\right)^{-}$such that (b) $\langle[f], g\rangle$ $=1$ and (c) $[f] \perp\left(C_{0}(G) \cap H_{P}^{\infty}(G)\right)^{-}$. From (a) and (c), we have $[f]=0$. This contradicts (b). Hence the proof is complete.

We can prove the following proposition by using Lemma 3.2 and the ideas in the proof of (Glicksberg and Wik (1971), Theorem 1.1, p. 620).

Proposition 3.3. Let $G$ be a nondiscrete LCA group whose dual $\hat{G}$ is algebraically ordered. Let $P$ be a subsemigroup of $\hat{G}$ with the $(A O)$-condition such that it is not dense in $\hat{G}$. Let $T$ be a linear operator on $H_{P}^{\infty}(G)$ such that it is continuous with respet to the weak* topology and commutes with translations. Then there exists a bounded regular measure $\mu$ on $G$ such that $T g=g * \mu$ for $g \in H_{P}^{\infty}(G)$.

Proof. Since $\left(L^{1}(G) / H_{(-P)}^{1}(G)\right)^{*} \cong H_{P}^{\infty}(G)$ and $T$ is continuous with respect to the weak* topology, there exists a multiplier $S$ on $L^{1}(G) / H_{(-P)}^{1}(G)$ such that $S^{*}=T$. For $g \in C_{0}(G) \cap H_{P}^{\infty}(G)$ and $x \in G$, we have $\left\|T g-\tau_{x} T g\right\|_{\infty}<$ $\|T\|\left\|g-\tau_{x} g\right\|_{\infty}$. Hence $T g$ is a bounded uniformly continuous function for each $g \in C_{0}(G) \cap H_{P}^{\infty}(G)$. We define a bounded linear functional $A$ on $C_{0}(G)$ $\cap H_{P}^{\infty}(G)$ as follows:

$$
A(g)=T g(0)
$$

Then, by the Hahn-Banach and the Riesz theorem, there exists a measure $\mu$ in $M(G)$ such that

$$
T g(x)=g * \mu(x) \text { for } g \in C_{0}(G) \cap H_{P}^{\infty}(G) \text { and } x \in G .
$$

Hence we have

$$
\begin{aligned}
& \langle g, S([f])-[f] * \mu\rangle=\langle T g,[f]\rangle-\langle g * \mu,[f]\rangle \\
& \quad=0 \text { for }[f] \in L^{1}(G) / H_{(-P)}^{1}(G) \text { and } g \in C_{0}(G) \cap H_{P}^{\infty}(G) .
\end{aligned}
$$


Hence, by Lemma 3.2, we have

$$
S([f])=[f] * \mu \text { for }[f] \in L^{1}(G) / H_{(-P)}^{1}(G) .
$$

Therefore $T g=g * \mu$ for $g \in H_{P}^{\infty}(G)$. This completes the proof.

We denote by $A P(G)$ the space of all almost periodic functions on $G$. We can identify $A P(G)$ with $C(\bar{G})$, where $\bar{G}$ denotes the Bohr compactification of $G$. Since $A P(G)$ is a translation invariant closed subspace of $L^{\infty}(G)$ containing constant functions, we can prove the following lemma by the same method in (Power (1977), Theorem 4, p. 74).

LEMMA 3.4. Let $m$ be the invariant mean on $A P(G)$ defined by $\int_{\bar{G}} f(\bar{x}) d \bar{x}$ for $f \in A P(G)$. Then there exists an invariant mean $M$ on $L^{\infty}(G)$ which is an extension of $m$.

Proposition 3.5. Let $G$ be a nondiscrete, noncompact $L C A$ group whose dual $\hat{G}$ is algebraically ordered. Let $P$ be a subsemigroup of $\hat{G}$ with the $(A O)$-condition such that it is not dense in $\hat{G}$. Then there exists a multiplier $T$ on $H_{P}^{\infty}(G)$ such that it is not given by convolution with a bounded regular measure on $G$.

Proof. Let $M$ be the invariant mean on $L^{\infty}(G)$ in Lemma 3.4. We define an operator on $H_{P}^{\infty}(G)$ as follows:

$$
T f(x)=M(f) \text { for } f \in H_{P}^{\infty}(G) \text { and } x \in G .
$$

Then $T$ is a multiplier on $H_{P}^{\infty}(G)$. Suppose $T f=f * \mu$ for some $\mu \in M(G)$. Let $\gamma$ be in $P \backslash\{0\}$. Then $T(\gamma)(x)=M(\gamma)=m(\gamma)=0$. On the other hand, $\gamma * \mu(x)$ $=\int_{G}(x-y, \gamma) d \mu(y)=(x, \gamma) \hat{\mu}(\gamma)$. Hence we have $\hat{\mu}(\gamma)=0$ on $P \backslash\{0\}$. Since $\hat{G}$ is not discrete, 0 is an accumulation point of $P \backslash\{0\}$. Hence we have $\hat{\mu}(0)=0$. On the other hand, since $\hat{G}$ is not discrete, constant functions belong to $H_{P}^{\infty}(G)$. Hence we have $T(1)(x)=M(1)=1$. This is a contradiction.

\section{Some multiplier on $C_{0}(G) / C_{0}(G) \cap H_{(-P)}^{\infty}(G)$.}

Let $\beta$ be the canonical map from $C_{0}(G)$ onto $C_{0}(G) / C_{0}(G) \cap H_{(-P)}^{\infty}(G)$. It is well known that a multiplier on $C_{0}(G)$ is given by convolution with a bounded regular measure on $G$ (Larsen (1971), Theorem 3.3.2, p. 74). Hence, for each multiplier $S^{\prime}$ on $C_{0}(G)$, there exists a multiplier $S$ on $C_{0}(G) / C_{0}(G) \cap H_{(-P)}^{\infty}(G)$ such that $\beta \circ S^{\prime}=S \circ \beta$. In this section, we prove that there exists a multiplier $S$ on $C_{0}(G) C_{0}(G) \cap H_{(-P)}(G)$ such that $S \circ \beta \neq \beta \circ S^{\prime}$ for every multiplier $S^{\prime}$ on $C_{0}(G)$ (see diagram). 


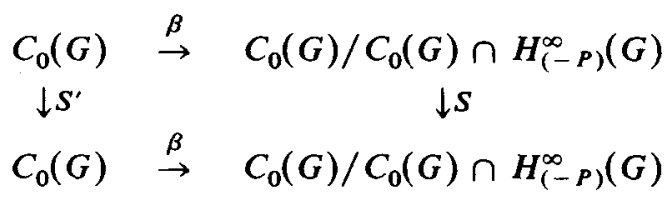

Definition 4.1. A bounded linear operator $T$ on $C_{0}(G) / C_{0}(G) \cap H_{(-P)}^{\infty}(G)$ is called a multiplier if it commutes with translations.

Proposition 4.2. Let $G$ be a LCA group such that $\hat{G}$ is algebraically ordered. Let $P$ be a subsemigroup of $\hat{G}$ with the $(A O)$-condition such that it is not dense in $\hat{G}$. Then we have $M_{P}^{a}(G) \cong\left(C_{0}(G) / C_{0}(G) \cap H_{(-P)}^{\infty}(G)\right)^{*}$.

Proof. Let $f$ be a function in $C_{0}(G) \cap H_{(-P)}(G)$ and $\mu$ a measure in $M_{P}^{a}(G)$. For each neighborhood $V$ of 0 in $G$ with the compact closure, we choose a nonnegative continuous function $g_{V} \operatorname{such}$ that $\operatorname{supp}\left(g_{V}\right) \subset V$ and $\int_{G} g_{V}(x) d x=$ 1. Then we have $\lim _{V} g_{V} * \mu=\mu$ in the weak* topology. Therefore, since $g_{V} * \mu \in H_{P}^{1}(G)$ and $f(-x) \in H_{P}^{\infty}(G)$, we have

$$
\begin{aligned}
\langle f, \mu\rangle & =\int_{G} f(-x) d \mu(x) \\
& =\lim _{V} \int_{G} f(-x) g_{V} * \mu(x) d x \\
& =0 .
\end{aligned}
$$

Thus $M_{P}^{a}(G)$ is included in $\left(C_{0}(G) \cap H_{(-P)}^{\infty}(G)\right)^{\perp}$.

Conversely, let $\mu$ be a measure in $M(G)$ such that $\mu \perp C_{0}(G) \cap H_{(-P)}^{\infty}(G)$. By Lemma 3.2, for each $\gamma_{0} \in(-P) \backslash\{0\}$, there exists a net $\left\{k_{\alpha}\right\}$ in $C_{0}(G) \cap$ $H_{(-P)}^{\infty}(G)$ such that $\lim _{\alpha} k_{\alpha}=\gamma_{0}$ in the weak* topology. Let $g$ be a function in $L^{1}(G)$ such that $\hat{g}\left(\gamma_{0}\right)=1$. For $\varepsilon>0$, since $g * \mu \in L^{1}(G)$, there exists $k_{\beta} \in$ $\left\{k_{\alpha}\right\}$ such that

$$
\left|\left\langle k_{\beta}, g * \mu\right\rangle-\left\langle\gamma_{0}, g * \mu\right\rangle\right|<\varepsilon
$$

On the other hand, since $k_{\beta} * g \in C_{0}(G) \cap H_{(-P)}(G)$, we have

$$
\left\langle k_{\beta}, g * \mu\right\rangle=\left\langle k_{\beta} * g, \mu\right\rangle=0 \text {. }
$$

Hence we have

$$
\left|\left\langle\gamma_{0}, \mu\right\rangle\right|=\left|\left\langle\gamma_{0}, g * \mu\right\rangle\right|\langle\varepsilon .
$$

Since $\varepsilon(>0)$ is arbitrary, $\mu$ belongs to $M_{P}^{a}(G)$. Therefore we have

$$
\left(C_{0}(G) \cap H_{(-P)}^{\infty}(G)\right)^{\perp} \subset M_{P}^{a}(G) .
$$

This completes the proof. 
THEOREM 4.3. Let $G$ be a nondiscrete LCA group such that $\hat{G}$ is algebraically ordered. Let $P$ be a subsemigroup of $\hat{G}$ with the $(A O)$-condition such that it is not dense in $\hat{G}$. Then there exists a linear operator $T$ on $M_{P}^{a}(G)$ as follows:

(i) $T$ is continuous with respect to the weak* topology,

(ii) $T$ commutes with translations,

(iii) $T$ is not given by convolution with a bounded regular measure on $G$.

Proof. From (Yamaguchi (1980), Theorem 11), there exists a multiplier $T_{\Phi}$ on $H_{P}^{1}(G)$ with the following properties:

(a) $T_{\Phi}(f)^{\wedge}=\Phi \hat{f}$ for $f \in H_{P}^{1}(G)$,

(b) $T_{\Phi}$ is not given by convolution with a bounded regular measure on $G$, where $\Phi$ is a bounded continuous function on $P^{0}$. We define a bounded Borel measurable function $\Phi^{\prime}$ on $\hat{G}$ as follows:

$$
\Phi^{\prime}(\gamma)= \begin{cases}\Phi(\gamma) & \text { for } \gamma \in P^{0} \\ 0 & \text { otherwise. }\end{cases}
$$

For $\mu \in M_{P}^{a}(G), \Phi^{\prime} \hat{\mu}$ belongs to $M_{P}^{a}(G)^{\hat{x}}$. Indeed, let $\gamma_{i}$ be in $\hat{G}$ and $c_{i} \in \mathbf{C}$ (complex numbers) $(i=1,2, \ldots, n)$. For $\varepsilon>0$, we choose a function $f$ in $L^{1}(G)$ such that $\|f\|_{1}<1+\varepsilon$ and $\hat{f}\left(\gamma_{i}\right)=1 \quad(i=1,2, \ldots, n)$. Put $q(x)=$ $\sum_{1}^{n} c_{i}\left(x, \gamma_{i}\right)$. Then, since $f * \mu \in H_{P}^{\prime}(G)$, we have

$$
\begin{aligned}
\left|\sum_{1}^{n} c_{i} \Phi^{\prime}\left(\gamma_{i}\right) \hat{\mu}\left(\gamma_{i}\right)\right| & =\left|\sum_{1}^{n} c_{i} \Phi^{\prime}\left(\gamma_{i}\right)(f * \mu)^{\wedge}\left(\gamma_{i}\right)\right| \\
& =\left|\sum_{1}^{n} c_{i} T_{\Phi}(f * f)^{\wedge}\left(\gamma_{i}\right)\right| \\
& \leqslant\|q\|_{\infty}\left\|T_{\Phi}(f * \mu)\right\|_{1} \\
& \leqslant(1+\varepsilon)\left\|T_{\Phi}\right\|\|\mu\|\|q\|_{\infty} .
\end{aligned}
$$

Since $\varepsilon(>0)$ is arbitrary, we haves

$$
\left|\sum_{1}^{n} c_{i} \Phi^{\prime}\left(\gamma_{i}\right) \hat{\mu}\left(\gamma_{i}\right)\right| \leqslant\left\|T_{\Phi}\right\|\|\mu\|\|q\|_{\infty} .
$$

Hence, by Bochner-Eberlein's theorem, $\Phi^{\prime} \hat{\mu}$ belongs to $M_{P}^{a}(G)^{\wedge}$.

Let $T$ be a linear operator on $M_{P}^{a}(G)$ such that $T(\mu)^{n}=\Phi^{\prime} \hat{\mu}$. Then, by the closed graph theorem, $T$ is continuous with respect to the norm topology. Since $T_{\Phi}$ is not given by convolution with a bounded regular measure on $G$, (iii) is satisfied. It is trivial that $T$ commutes with translations.

Next we prove that $T$ is continuous with respect to the weak* topology. Let $\mu$ be a measure in $M_{P}^{a}(G)$ and $\left\{\mu_{\alpha}\right\}_{\alpha \in \Lambda}$ a net in $M_{P}^{a}(G)$ such that $\left\|\mu_{\alpha}\right\|<C$ and $\mu_{\alpha}$ converges weak ${ }^{*}$ to $\mu$, where $C$ is a positive constant such that $\|\mu\| \leqslant C$. Let $f$ be a function in $C_{0}(G)$ and $\varepsilon>0$. Since $L^{1}(\hat{G})^{\wedge}$ is dense in $C_{0}(G)$, there exists a 
function $g$ in $C_{c}(\hat{G})$ such that $\|f-\hat{g}\|_{\infty}<\varepsilon / 4(1+C\|T\|)$. Put $F(\gamma)=$ $\Phi^{\prime}(\gamma) g(\gamma)$. Then $F$ belongs to $L^{1}(\hat{G})$. Hence there exists $\alpha_{0} \in \Lambda$ such that

$$
\left|\int_{G} \hat{F}(x) d \mu_{\alpha}(x)-\int_{G} \hat{F}(x) d \mu(x)\right|<\frac{\varepsilon}{2} \quad \text { for } \alpha \geqslant \alpha_{0} .
$$

Therefore, if $\alpha \geqslant \alpha_{0}$, we have

$$
\begin{aligned}
\mid \int_{G} f(x) d T\left(\mu_{\alpha}\right)(x)- & \int_{G} f(x) d T(\mu)(x)|<| \int_{G}(f(x)-\hat{g}(x)) d T\left(\mu_{\alpha}\right)(x) \mid \\
& +\left|\int_{G} \hat{g}(x) d T\left(\mu_{\alpha}\right)(x)-\int_{G} \hat{g}(x) d T(\mu)(x)\right| \\
& +\left|\int_{G}(\hat{g}(x)-f(x)) d T(\mu)(x)\right| \\
< & \varepsilon / 4(1+C\|T\|) \times\left\|T\left(\mu_{\alpha}\right)\right\|+\varepsilon / 4(1+C\|T\|) \times\|T(\mu)\| \\
& +\left|\int_{G} \hat{g}(x) d T\left(\mu_{\alpha}\right)(x)-\int_{G} \hat{g}(x) d T(\mu)(x)\right| \\
< & \frac{\varepsilon}{2}+\left|\int_{\hat{G}} G\left(\mu_{\alpha}\right)^{-}(\gamma) g(\gamma) d \gamma-\int_{\hat{G}} T(\mu)^{\wedge}(\gamma) g(\gamma) d \gamma\right| \\
= & \frac{\varepsilon}{2}+\left|\int_{\hat{G}} F(\gamma) \hat{\mu}_{\alpha}(\gamma) d \gamma-\int_{\hat{G}} F(\gamma) \hat{\mu}(\gamma) d \gamma\right| \\
= & \frac{\varepsilon}{2}+\left|\int_{G} \hat{F}(x) d \mu_{\alpha}(x)-\int_{G} \hat{F}(x) d \mu(x)\right| \\
< & \varepsilon .
\end{aligned}
$$

That is, $T\left(\mu_{\alpha}\right)$ converges to $T(\mu)$ in the weak* topology. Hence, by (Larsen (1971), Appendix D.4.2 Theorem), $T$ is continuous with respect to the weak* topology. This completes the proof of Theorem 4.3.

Remark 4.4. By (Koshi and Yamaguchi (1979), Theorem 1 and Theorem 2, pp. 295, 296), $M_{P}^{a}(G)=H_{P}^{1}(G)$ if and only if $G$ is one of the following:

$$
\text { (1) } G=T \oplus D, \quad \text { (2) } G=R \oplus D,
$$

where $D$ is a discrete abelian group whose dual is torsion-free or $D=\{0\}$.

Suppose $M_{P}^{a}(G)=H_{P}^{1}(G)$, then for each multiplier $S$ on $M_{P}^{a}(G)$, there exists a bounded continuous function $\psi$ on $P^{0}$ such that $S(f)^{\wedge}=\psi \hat{f}$. Hence, in this case, we can verify that every multiplier on $M_{P}^{a}(G)$ is continuous with respect to the weak* topology by the same method used in the proof of Theorem 4.3. However, if $H_{P}^{\prime}(G) \varsubsetneqq M_{P}^{a}(G)$, there exists a multiplier on $M_{P}^{a}(G)$ such that it is not continuous with respect to the weak* topology. Indeed, we define a linear operator $S$ from $M_{P}^{a}(G)$ into $M(G)$ as follows:

$$
S(\mu)=\mu_{s} \text {, where } \mu_{s} \text { is the singular part of } \mu .
$$


Then, by (Doss (1968), Lemma 1, p. 258), $\mu_{s}$ belongs to $M_{P}^{a}(G)$ if $\mu \in M_{P}^{a}(G)$. Hence $S$ is a multiplier on $M_{P}^{a}(G)$. Since $H_{P}^{1} \subsetneq M_{P}^{a}(G)$, there exists a nonzero measure $\mu_{0}$ in $M_{P}^{a}(G)$ which is singular with respect to the Haar measure on $G$. Let $\dot{V}$ be the net consisting of all neighborhoods of 0 in $\hat{G}$. For each $V \in \dot{V}$, let $f_{V}$ be a nonnegative continuous function on $G$ with the compact support such that $\operatorname{supp}\left(f_{V}\right) \subset V$ and $\int_{G} f_{V}(x) d x=1$. Then $f_{V} * \mu_{0}$ converges to $\mu_{0}$ in the weak* topology. But, since $f_{V} * \mu_{0} \in H_{P}^{1}(G), S\left(f_{V^{*}} \mu_{0}\right)(=0)$ does not converge to $\mu_{0}$ in the weak* topology.

COROLLARY 4.5. Let $G$ be a nondiscrete LCA group such that $\hat{G}$ is algebraically ordered. Let $P$ be a subsemigroup of $\hat{G}$ with the $(A O)$-condition such that it is not dense in $G$. Then there exists a multiplier $S$ on $C_{0}(G) / C_{0}(G) \cap H_{(-P)}^{\infty}(G)$ such that it is not given by convolution with a bounded regular measure on $G$.

Proof. Let $T$ be the multiplier on $M_{P}^{a}(G)$ obtained in Theorem 4.3, and put $S=T^{*}$. Then, since $T$ is continuous with respect to the weak ${ }^{*}$ topology, $S$ is a multiplier on $C_{0}(G) / C_{0}(G) \cap H_{(-P)}^{\infty}(G)$. Suppose there exists a measure $\mu \in$ $M(G)$ such that $S([f])=[f] * \mu$ for $[f] \in C_{0}(G) / C_{0}(G) \cap H_{(-P)}^{\infty}(G)$. Then we have $T(\nu)=\nu * \mu$ for $\nu \in M_{P}^{a}(G)$. This is a contradiction.

COROLlary 4.6. Let $G$ and $P$ be as in Corollary 4.5. Let $\beta$ be the canonical map from $C_{0}(G)$ onto $C_{0}(G) / C_{0}(G) \cap H_{(-P)}^{\infty}(G)$. Then there exists a multiplier $S$ on $C_{0}(G) / C_{0}(G) \cap H_{(-P)}^{\infty}(G)$ such that $S \circ \beta \neq \beta \circ S^{\prime}$ for every multiplier $S^{\prime}$ on $C_{0}(G)$.

Proof. Let $S$ be the multiplier on $C_{0}(G) / C_{0}(G) \cap H_{(-P)}^{\infty}(G)$ obtained in Corollary 4.5. Suppose there exists a multiplier $S^{\prime}$ on $C_{0}(G)$ such that $S \circ \beta=$ $\beta \circ S^{\prime}$. Then there exists a measure $\mu \in M(G)$ such that $S^{\prime}(f)=f * \mu$ for $f \in C_{0}(G)$. Hence, since $g * \mu \in C_{0}(G) \cap H_{(-P)}^{\infty}(G)$ for $g \in C_{0}(G) \cap$ $H_{(-P)}^{\infty}(G), S$ is given by $S([f])=[f] * \mu$ for $[f] \in C_{0}(G) / C_{0}(G) \cap H_{(-P)}^{\infty}(G)$. This contradicts the fact that $S$ is not given by convolution with a measure.

Finally I wish to express my thanks to the referee for his valuable advice.

\section{References}

F. Birtel (1966), Function algebra, (Scott, Foresman and Company, Glenview, Illinois).

R. Doss (1968), 'On measures with small transforms', Pacific J. Math. 26, 257-263. (1970), 'On analytic contractions in $L^{P}(G)$ ', Math. Z. 114, 59-65.

G. I. Gaudry (1968), ' $H^{p}$ multipliers and inequality of Hardy and Littlewood', J. Austral. Math. Soc. 10, 23-32. 
I. Glicksberg and I. Wik (1971), 'Multipliers of quotient of $L^{1}$ ', Pacific J. Math. 38, 619-624.

E. Hewitt and K. A. Ross (1970), Abstract harmonic analysis II, (Springer-Verlag, Berlin-HeidelbergNew York).

S. Koshi and H. Yamaguchi (1979), 'The F. and M. Riesz theorem and group structures', Hokkaido Math. J. 8, 294-299.

R. Larsen (1971), Introduction to the theory of multipliers, (Springer-Verlag, Berlin-Heidelberg-New York).

H. Otaki (1977), 'A relation between the F. and M. Riesz theorem and the structure of LCA groups', Hokkaido Math. J. 6, 306-312,

S. Power (1977), ' $H^{\infty}+A P$ is closed', Proc. Amer. Math. Soc. 65, 73-76,

W. Rudin (1962), Fourier analysis on groups, (Interscience, New York).

H. Yamaguchi (1980), 'Some multipliers on $H_{P}^{1}(G)$ ', J. Austral. Math. Soc. (Ser. A) 29, 52-60.

Department of Mathematics

Hokkaido University

Sapporo

Japan
Current address: Department of Mathematics Josai University Sakado, Saitama

Japan 